with $>2$ exposures. Infectious diseases were by far the most common outcomes studied (44\%): blood-borne viruses alone accounted for $33 \%$ of all data points. The next most common outcomes were external causes, injury, and poisoning (16\%) and mental disorders (14\%): together with infections, these categories accounted for $73 \%$ of all data points.

Conclusion Existing research on the health of people experiencing multiple forms of social exclusion is dominated by cross-sectional studies examining a relatively limited set of exposures and outcomes. The lack of data on more than two exposures in combination; self-rated health or quality of life; or non-communicable diseases (e.g. cardiovascular disease and cancer) suggests that research to date may not reflect the true burden of ill-health in these populations. Limitations include restriction to peer-reviewed studies and risk of publication bias.

\section{OP43 A CASE CONTROL STUDY OF SOCIAL DETERMINANTS OF TUBERCULOSIS RISK IN WHITE UK-BORN ADULTS IN ENGLAND}

'P Nguipdop Djomo*, 'LC Rodrigues, ${ }^{1} \mathrm{PG}$ Smith, ${ }^{2}$ A Abubakar, ${ }^{1} \mathrm{P}$ Mangtani. 'Infectious Disease Epidemiology, London School of Hygiene and Tropical Medicine, London, UK; ${ }^{2}$ Institute for Global Health, University College London, London, UK

\subsection{6/jech-2019-SSMabstracts.44}

Background Tuberculosis (TB) remains a public health problem in the UK-born population of England, including among young adults. Ecological studies indicate that deprivation is an important risk factor for $\mathrm{TB}$, but there are few recent individual-level studies in high income countries that have investigated the association between poverty-related social determinants of health inequality $(\mathrm{SDH})$ and $\mathrm{TB}$.

Our objective was to measure the association between individual socio-economic status and social determinants of health, and $\mathrm{TB}$, taking into account the clustering of social risk factors in individuals, and to estimate the potential population impact on TB rates.

Methods Secondary analyses of a nationwide case-control study conducted among UK-born White adults aged 23 to 38 years at diagnosis of their first TB episode, and randomly selected age and sex frequency-matched community controls. Data on some SDH (education, household overcrowding, tobacco smoking, alcohol use, drugs use, and history of homelessness and prison) were collected in face-to-face interviews. Statistical analyses, using logistic regression models, was informed by a theoretical causal framework (Directed Acyclic Graph) of plausible inter-relationships between the measured social factors.

Results Overall, 681 TB cases and 1183 controls were recruited. The risk of $\mathrm{TB}$ was about four times higher in subjects whose formal education was up to GCSE O-levels or less compared to those with at least a university degree $(\mathrm{OR}=3.94 ; 95 \% \mathrm{CI}: 2.74 ; 5.67)$, after controlling for other TB risk factors (age, sex, BCG vaccination and stays in Africa or Asia for ${ }^{3} 3$ months). After simultaneously adjusting for these risk factors and all measured social determinants, higher TB risk was also independently associated with tobacco smoking, use of drugs (especially injectable drugs - OR=5.67; 95\% CI: $2.68 ; 11.98$ ), history of homelessness and deprivation in the area of residence. Population Attributable Fraction (PAF) estimates suggested that tobacco use and class-A drug use were, respectively, responsible for $18 \%$ and $15 \%$ of $\mathrm{TB}$ cases in the target population.

Conclusion The results provide insight into some of the mechanisms through which deprivation increases the risk of $\mathrm{TB}$ in the general population in England and support the argument for improved approaches to TB control efforts, such as integrated health and social services in high-risk young adult populations.

\section{OP44 DOES ETHNIC-RACIAL IDENTITY MODIFY THE EFFECTS OF RACISM ON AUSTRALIAN ABORIGINAL CHILDREN SOCIO-EMOTIONAL WELLBEING?}

DM Macedo*, ${ }^{2}$ LG Smithers, ${ }^{3}$ R Roberts, ${ }^{2}$ DG Haag, 'LM Jamieson. 'S School of Dentistry, University of Adelaide, Adelaide, Australia; ${ }^{2}$ School of Public Health, University of Adelaide, Adelaide, Australia; ${ }^{3}$ School of Psychology, University of Adelaide, Adelaide, Australia

\subsection{6/jech-2019-SSMabstracts.45}

Background Racism impacts the health and wellbeing of racial minorities across the lifespan. Ethnic-racial identity (ERI) has been suggested as a protective factor against adversity on racial minorities' wellbeing, however, findings vary between ethnic-racial groups. Research in the Aboriginal Australian context is still limited. The present study tests ERI affirmation as an effect-modifier of the longitudinal association between racism and Aboriginal Australian children's socio-emotional wellbeing (SEWB). Effect-sizes are hypothesized to be smaller among children with higher ERI affirmation.

Methods Children $(n=408)$ from the K-Cohort of the Longitudinal Study of Indigenous Children (LSIC) were included in the analysis. Data were collected through questionnaire-guided interviews in two time points, when children were 7-10 years and 9-12 years. Children's racism experience, SEWB (assessed by the Strengths and Difficulties Questionnaire), and confounders were reported by caregivers. Information on ERI affirmation was provided through child self-report. Analyses were conducted in Stata 14. Multiple imputation with chained equations was used to address potential bias due to missing data. Poisson regression with robust errors estimated adjusted Risk Ratios (RRa) for the longitudinal effect of racism on different domains of child SEWB. For the effect-measure modification analysis, $\mathrm{RR}_{\mathrm{a}}$ were obtained for the different stratum of the exposure (racism) and the effect-modifier (ERI affirmation). The direction of the modification was indicated by the Relative Excess Risk due to Interaction (RERI) on the additive scale.

Results Children exposed to racism and with low ERI affirmation were at increased risk of increased socio-emotional difficulties, especially for hyperactive behaviour ( $\mathrm{RRa} 2.16$, 95\% CI 1.00, 4.67), conduct problems (RRa 1.76, 95\% CI 0.71 , 3.83), and total difficulties ( $R R a 1.94,95 \%$ CI $0.92,4.11$ ), although the $95 \%$ confidence intervals were wide. Positive effect-measure modification was found in these domains, with respective RERIs of $1.08,1.39$, and 1.14 . Contrary to our hypothesis, children with high ERI affirmation were at increased risk of peer problems (RRa 1.80, 95\% CI 0.83 , 3.90). A negative effect-measure modification was found in this domain (RERI: -0.75).

Conclusion Results indicate that promoting ERI affirmation among Aboriginal Australian children might assist to mitigate the effects of racism on specific domains of child SEWB. 